\title{
La Réception Visuelle du Texte: Analyse du Poème Familiale de Jacques Prévert Selon les Théories de la Réception
}

\author{
Khaled Younes* \\ Département des Langues Modernes, Université de Yarmouk
}

Received on: 18-2-2020

Accepted on: 7-5-2020

\section{Résumé}

Cet article représente une analyse du poème Familiale selon les théories de la réception. L'écart esthétique créé par le texte attire le lecteur et l'incite à poursuivre la lecture. L'influence du contexte socioculturel sur le lecteur, et la projection du vécu de ce dernier sur le texte se révèlent importantes pour comprendre et analyser le texte. Toutefois, pour l'interprétation du texte, le processus dinteraction entre le texte et le lecteur joue un rôle aussi important. Cet article a démontré qu'une grande partie de ce processus d'interaction se fait à travers la réception visuelle: le texte stimule l'intelligence visuelle-spatiale chez le lecteur, et le lecteur, de son côté, réagit en utilisant cette capacité pour pouvoir le comprendre et l'interpréter.

Mots-clés: Analyse, Interprétation, Familiale, Texte, Visuelle, Réception.

\section{Visual Reception of the Text: Analysis of Jacques Prévert's Poem "Familiale" according to the Reception Theories}

\begin{abstract}
This article presents an analysis of the poem "FAMILIALE" according to the reception theories. The aesthetic gap created by the text attracts the reader and urges him to continue reading. The influence of the socio-cultural context on the reader, and the projection of the latter's experience on the text appear to be important for understanding and analyzing the text. However, in interpreting the text, the process of interaction between the text and the reader plays an important role. This article shows that a large part of this interaction process takes place through visual reception: the text stimulates the reader's visual-spatial intelligence, and the reader reacts by using this capacity to be able to understand and interpret it.
\end{abstract}

Keywords: Analysis, Interpretation, Familiale, Text, Visual, Reception.

\section{Introduction}

Beaucoup de textes poétiques, surtout de la poésie contemporaine, nous paraissent simples et directs. Certes, une simple lecture et sans concentration en est la raison. C'est donc une simplicité et une clarté de sens trompeuses, qui cachent une profondeur de sens qui ne peut être atteinte qu'après une lecture très

\footnotetext{
๑ 2021 JJMLL Publishers/Yarmouk University. All Rights Reserved.

${ }^{*}$ Doi.org/10.47012/jjmll.13.1.5

* Corresponding author: kfiy1972@gmail.com
} 
attentive que le lecteur doit effectuer pour interpréter le texte. Cela exactement ce qui nous est arrivé en lisant le poème intitulé "Familiale" de Jacques Prévert, et ce que nous entendons expliquer dans cet article.

Le texte Familiale est extrait d'une œuvre poétique intitulée "Paroles" (Prévert 1949). Jacques Prévert avait écrit ce poème juste après la fin de la deuxième guerre mondiale, en exprimant son opinion et son expérience vécue pendant la guerre. La question qui se pose maintenant est la suivante: notre lecture et notre réception du même texte aujourd'hui seraient-elles différentes? L'un des domaines que les théories de la réception étudient est l'influence du contexte socioculturel du lecteur sur sa compréhension du texte. Mais, il y a encore d'autres moyens qui aident le lecteur à comprendre le texte: ses expériences, ses lectures antérieures, son interaction avec le texte. Tous ces moyens aident le lecteur à comprendre le texte et en affectent sa réception, ce que nous avons l'intention d'éclaircir dans notre article.

Notre objectif sera donc d'expliquer la pratique de notre lecture et le résultat de notre réception du poème Familiale en répondant aux questions suivantes: quelles émotions avons-nous vécues et quelle était notre réaction en lisant ce texte? Quel effet ce texte a-t-il eu sur nous? Et en conséquence, comment avonsnous interprété ce texte?

Pour arriver à notre objectif et répondre aux questions posées, nous nous appuierons sur les théories de la réception qui s'accordent toutes sur le principe d'éloigner les études littéraires de l'analyse axée sur la relation auteur/texte vers une analyse basée sur le rapport texte/lecteur, en donnant plus dimportance au lecteur en particulier. Ce qui sera très utile pour notre article qui se concentrera sur ce principe.

Bien que les penseurs des théories de la réception soient nombreux et que chacun ait ajouté un point de vue important et apprécié, pour l'objectif de notre recherche nous nous concentrerons en particulier sur les idées de Jauss (1978), Iser (1976) Ricœur (1986) et Fish (1980). Lorsque nous avons étudié les théories de ces penseurs concernant la posture du lecteur vis-à-vis du texte, nous avons trouvé que leurs idées correspondaient exactement à ce que nous avons expérimenté pendant notre lecture du texte Familiale.

Or, dans un premier temps, nous présenterons un survol des idées de ces quatre penseurs, mais, ce survol ne suivra pas un ordre chronologique, car nous allons plutôt regrouper les différents points de vue en deux tendances opposées: une première tendance, représentée par Jauss et Fish, qui considère le lecteur comme faisant partie d'un groupe de lecteurs, et qui attribuent une grande importance au contexte socioculturel auquel le lecteur appartient, et une deuxième tendance qui considère l'acte de lecture comme un échange entre un texte et un individu ayant d'émotions et d'influences, et celle-ci est représentée par Iser et Ricœur.

Dans un deuxième temps, et à la lumière des théories de ces critiques littéraires, nous présenterons le fruit de notre compréhension et notre lecture du texte, en montrant comment cette lecture se déroulera. A chaque partie de notre analyse du texte, nous ferons recours aux théories de la réception pour justifier notre analyse. Nous présenterons enfin le résultat de notre travail, ainsi que notre point de vue concernant la relation texte/ lecteur. 
La Réception Visuelle du Texte: Analyse du Poème Familiale de Jacques Prévert Selon les Théories de la Réception

\section{Texte et théories de la réception}

\subsection{Le lecteur et le contexte socioculturel}

Selon Jauss, le lecteur est considéré comme un fragment du contexte socioculturel d'où il a déjà ses connaissances préalables. Jauss a construit sa théorie autour du concept d'Horizon d'Attente qu'il définit comme étant un "système de relations objectivement formulable des attentes qui résulte pour chaque œuvre au moment de sa parution des présupposés du genre, de la forme et de la thématique d'œuvres connues auparavant et de l'opposition entre langue poétique et langue pratique, entre monde imaginaire et réalité quotidienne" (Jauss 1978, 54)

Ainsi, le lecteur reçoit et juge une œuvre littéraire en étant influencé par l'arrière-plan de l'expérience de sa vie quotidienne, de sa vision du monde- et de ses connaissances littéraires préexistantes qui créent chez lui son horizon d'attente de cette œuvre. Cet horizon d'attente peut être compris ici comme toutes les suppositions anticipées que le lecteur met sur une œuvre avant sa lecture. Mais cette œuvre littéraire peut, d'une part, s'harmoniser avec l'horizon d'attente du lecteur, en répondant aux suppositions déjà mises, et d'autre part, elle peut s'éloigner de cet horizon d'attente en créant alors un certain choc de réception, ce que Jauss appelle l'écart esthétique, qui oppose l'horizon littéraire du texte à la disposition d'esprit ou le code esthétique du lecteur qui conditionne la réception, nous offrant ainsi un moyen pour mesurer la qualité et la beauté d'une œuvre littéraire de sorte que plus cet écart entre l'horizon du texte et l'horizon d'attente du lecteur est grand, plus l'œuvre littéraire est appréciée et estimée (Jauss 1978, 59).

Fish donne une grande importance au contexte social. Il rejoint Jauss en considérant le lecteur en tant qu'appartenant à un groupe de lecteurs/ récepteurs qui se mettent tacitement d'accord sur les lignes selon lesquelles un texte sera interprété. L'interprétation d'un texte littéraire change à chaque fois que le lecteur lit ce texte, et ce changement dépend du contexte social dans lequel le lecteur vit au moment de la lecture: "Paradoxically, the exercise does not prove that the words can mean anything one likes, but that they always and only mean one thing, although that one thing is not always the same" (Fish 1980, 275). Ainsi, le lecteur agit vis-à-vis du texte en utilisant une certaine mémoire collective qu'il partage avec d'autres lecteurs appartenant à la même "communauté interprétative" ou "communauté de lecteurs", alors que le lecteur "peut appartenir à plusieurs communautés en même temps ou passer d'une communauté à l'autre (...). Cependant, c'est son appartenance à une communauté qui détermine son rapport au texte" (Cools 2011, 51).

\subsection{Le rapport entre le texte et le lecteur en tant qu'un individu}

Iser, quant à lui, voit la lecture comme un rapport dialogique qui s'établit entre le lecteur et le texte pour atteindre à un moment d'entente et de rencontre entre les deux. Pour Iser, la lecture d'un texte est un événement: "Etant donné que le rapport entre le texte et le lecteur se réalise par une information en retour sur les effets produits sur le lecteur au cours du processus d'action, ce processus se développe en tant que processus constant de réalisation. Le processus se déroule grâce aux signifiés que le lecteur produit et transforme. Dès lors le contexte événementiel acquiert le caractère d'une situation ouverte qui est toujours à la fois concrète et susceptible de se transformer" (Iser 1976, 126) 
L'acte de lecture est considéré ici comme une phénoménologie propre au lecteur, et non plus un processus conditionné socialement (Iser 1976, 144). Iser considère la lecture comme une interaction intime entre texte et lecteur, le lecteur pose des questions, interroge le texte et s'interroge lui-même sur le texte. Ce dernier, de son coté, offre des orientations qui permettent au lecteur d'interpréter le sens. Mais, dans ce processus d'interaction entre le texte et le lecteur, ce dernier sera guidé par le texte, qui lui offre des indices et lui laisse des blancs, ce que Iser appelle "les indéterminations" que le lecteur doit remplir en utilisant son répertoire de connaissances et d'expériences précédentes, en particulier ses lectures antérieures (Iser 1976, 273-274). Le contexte socioculturel n'a donc plus dimportance. Par contre, Iser attribue une grande importance à la culture individuelle du lecteur, à travers ses expériences personnelles et ses lectures préalables, qui aident ce dernier à combler les blancs du texte, ce qui aboutit enfin à la compréhension et à l'interprétation du texte. En même temps, Iser trouve que le texte évoque une réalité qui se définit partiellement par la structure sociale, mais cette réalité est vécue par un individu unique (Iser 1976, 274).

L'herméneutique de Ricœur nous offre une vision du texte en tant qu'un lieu de projection de la réalité du monde où nous vivons: "ce qui est à interpréter dans un texte, c'est une proposition de monde, le projet d'un monde que je pourrais habiter et où je pourrais projeter mes possibles les plus propres" (Ricœur 1986, 58). Pour Ricœur, la réception herméneutique d'un texte est un processus qui consiste, en premier lieu, à comprendre, en sachant que la compréhension est définie par Ricœur comme "la capacité de reprendre en soi-même le travail de structuration du texte", et en deuxième lieu, à expliquer, ça veut dire "l'opération de second degré greffée sur cette compréhension et consistant dans la mise au jour des codes sous-jacents à ce travail de structuration que le lecteur accompagne" (Ricœur 1986, 37). Alors, le sens est dans le texte, et le lecteur est invité à le trouver. Ce sont donc les éléments textuels, leur forme, leur mise en ordre et leur agencement qui nous aident à comprendre et puis à expliquer le texte (Cools 2011, 54).

Après ce résumé des pensées des théoriciens de la réception et de la lecture, nous montrerons dans la suite de notre article comment le processus de notre lecture du texte Familiale s'accorde avec toutes ces pensées, pour enfin arriver à notre propre interprétation du texte.

\section{L'interprétation du texte Familiale}

L'interaction entre le lecteur et le texte commence dès le début, quand le lecteur se trouve face à un blanc lorsqu'il lit le titre. Le poème s'est attribué un titre ambigu qui donne au lecteur à penser. Le terme "familiale" est un adjectif qualificatif dont le nom qualifié n'est pas mentionné. C'est donc au lecteur de deviner ce qui est entendu par familiale et il commence dès lors à interroger le texte et à anticiper la suite: s'agit-il d'une relation familiale? D'une scène familiale? Ou bien d'une histoire familiale? La réponse est évidemment à trouver petit à petit pendant la lecture du texte.

Dans les lignes suivantes, nous expliquerons les étapes du processus de notre lecture du texte sous deux titres principaux: l'image routinière de la guerre; et les relations guerre-affaires. 
La Réception Visuelle du Texte: Analyse du Poème Familiale de Jacques Prévert Selon les Théories de la Réception

\section{II.1 L'image routinière de la guerre}

La guerre dans le texte est représentée comme routinière, une chose normale et ordinaire dans le quotidien d'un peuple représenté par une famille composée de mère, père et fils. Cette image routinière de la guerre peut être conclue à travers trois indices donnés par le texte lui-même qui, comme nous l'avons vu selon Iser, nous laisse des blancs, des vides, et c'est à nous (lecteur) de les combler en décryptant les indices. Ce que nous analyserons dans les pages suivantes.

\subsubsection{La figure des parents indifférents face à la guerre}

En utilisant le temps du présent du verbe faire, le texte met le lecteur dans une scène:

\section{La mère fait du tricot \\ Le fils fait la guerre}

Les deux premiers vers du poème montrent la mère en faisant une activité quotidienne habituelle et routinière: le tricot. Tandis que le fils fait la guerre, une activité qui doit être bouleversante, inhabituelle, une exception dans la vie quotidienne d'une famille. A ce moment, le lecteur commence à construire ses hypothèses sur le texte en dépendant de ses expériences précédentes et de ses connaissances antérieures concernant les textes poétiques traitant la guerre, en attendant que ce texte évoque tous les termes du champ lexical de la guerre: dévastation, mort, malheur, tristesse, bouleversement, désordre...etc. Ainsi, l'horizon d'attente du lecteur se forme: si le fils fait la guerre, la mère doit être troublée, inquiétée et triste. Mais, le troisième vers vient rompre cette attente, en créant le premier écart esthétique dans le texte:

\section{Elle trouve ça tout naturel la mère}

Le lecteur commence à interroger le texte: la mère ne sinquiète-t-elle pas pour son fils? Tellement estelle indifférente envers la participation de son fils à la guerre qu'elle trouve cela tout naturel? Et le père peut-il être du même avis que la mère? Cette fois-ci, la réponse est à anticiper: puisque la mère, qui est censée avoir un caractère plus émotif que le père, trouve ça naturel, alors le père, lui aussi trouvera ça naturel. Les vers 4-9 nous confirment cette idée:

\section{Et le père qu'est-ce qu'il fait le père? \\ Il fait des affaires \\ Sa femme fait du tricot \\ Son fils la guerre \\ Lui des affaires \\ Il trouve ça tout naturel le père}

Donc, le père fait son activité quotidienne normale, son travail dans les affaires, comme la mère faisant son tricot, mais aussi, comme le fils faisant la guerre, ce que les parents trouvent "tout naturel". Linteraction entre l'œuvre et le lecteur continue, ce dernier continuant à interroger le texte: pourquoi cette froideur de sentiments et cette nonchalance de la part des parents? Y aurait-il un ton critique dans ces vers? 
A ce moment de la lecture, nous commençons à nous identifier dans le texte. Des images de notre vie quotidienne et de nos expériences dans le contexte social auquel nous appartenons commencent à émerger dans notre mémoire: dans ce coin du monde où nous vivons, nous entendons chaque jour des nouvelles de conflits, de dévastation, de catastrophes, de mort, de crimes de guerre, de massacres...etc. à tel point qu'avec le temps ces événements passent pour habituels et font partie de notre vie quotidienne. Une sorte de normalisation des relations avec la violence. Là, l'influence de notre contexte est claire sur notre réception de l'œuvre littéraire. Mais, ce qui nous intéresse ici c'est l'influence du contexte sur les émotions personnelles du lecteur en tant qu'un individu. Cela mène vers la projection du contexte social et de la réalité vécue sur le texte, et aide, par conséquent, à la compréhension et à l'interprétation du texte selon la vision du lecteur.

Cette figure des parents indifférents continue à décevoir le lecteur et rompt son horizon d'attente quand nous lisons la suite du texte:

\section{La guerre continue la mère continue elle \\ tricote \\ Le père continue il fait des affaires \\ Le fils est tué il ne continue plus}

Le fils est donc tué à la guerre. Le lecteur attend cette fois-ci que les parents réagissent. Ils doivent être chagrinés et bouleversés. Ils doivent exprimer leurs émotions, leur refus de la guerre. Mais, pour la deuxième fois, cette attente est rompue quand le lecteur lit:

\section{Le père et la mère vont au cimetière \\ Ils trouvent ça tout naturel le père et la \\ mère}

Le texte Familiale est donc un refus, un rejet, et une critique de la guerre, mais le texte critique également la mentalité du peuple qui ne réagit pas à la guerre. Le peuple s'est habitué à la violence. Il a tellement vu de malheur pendant la guerre que cette dernière est devenue une activité quotidienne habituelle, ordinaire et routinière, tout comme le tricot pour la mère et les affaires pour le père.

\subsubsection{La simplicité du langage}

Le deuxième indice remarquable dans le texte est le langage utilisé. C'est un langage simple et familier qui caractérise tout le poème. Dans certains passages, le lecteur a même l'impression que le texte utilise un langage infantile, comme par exemple dans les vers 4 et 5 :

$$
\begin{aligned}
& \text { Et le père qu'est-ce qu'il fait le père? } \\
& \text { Il fait des affaires }
\end{aligned}
$$

Et dans les vers 10-12:

$$
\begin{aligned}
& \text { Et le fils et le fils } \\
& \text { Qu'est-ce qu'il trouve le fils? }
\end{aligned}
$$




\section{La Réception Visuelle du Texte: Analyse du Poème Familiale de Jacques Prévert Selon les Théories de la Réception \\ Il ne trouve rien absolument rien le fils}

Le lecteur, et toujours pour combler les blancs dans le texte, se réfère à son répertoire des connaissances et des lectures préalables. Ainsi, ces vers précédents, avec leur contenu (répétition, interrogation...etc.) et aussi avec leur rythme, évoquent dans notre mémoire les comptines et les chansons que les enfants apprennent à l'école maternelle et pendant les premières années à l'école primaire. Ces comptines se caractérisent par la simplicité du langage et la répétition des énoncés qui aident les enfants à mémoriser les paroles, et qui se caractérisent également par l'abondance des phrases interrogatives accompagnées des réponses. Prenons comme exemple la comptine intitulée A la ferme:

Qui est blanche avec des taches?

C'est la vache, c'est la vache

Qui est doux comme un câlin

C'est le lapin, c'est le lapin

Qui galope et qui cavale?

C'est le cheval, c'est le cheval

(...)

Et aussi la comptine intitulée Les couleurs:

Les couleurs, les couleurs

Je les connais toutes par cœur

Les couleurs, les couleurs

Chantons-les...chantons-les tous en chœur

(...)

Une telle simplicité du langage dans le texte reflète, à notre avis, la simplicité de penser la guerre et la normalisation de son image dans la mentalité collective d'un peuple qui s'est familiarisé avec la violence, et face à laquelle il ne montre aucune réaction, ce que le texte critique implicitement.

\subsubsection{La répétition}

Le troisième indice offert par le texte est l'abondance de la répétition. Quand le lecteur continue à découvrir le texte, il remarque qu'il y a beaucoup de répétitions face auxquelles il ne reste pas indifférent. A notre avis, la répétition est le moyen par excellence utilisé dans le texte pour concrétiser l'image routinière de la guerre.

Certes il est très important ici d'expliquer et d'éclaircir les parties répétitives dans le texte, mais, dans le souci de ne pas être répétitif nous-même, nous trouvons que c'est mieux d'en parler amplement dans la partie suivante où la répétition est un élément important. 


\subsection{Les rapports guerre-affaires}

Revenons au début du texte. Quand le lecteur commence à lire Familiale, dès le début, il découvre un autre blanc à combler quand il remarque l'abondance de la répétition. Le lecteur s'interroge sur l'importance de toutes ces répétitions et leur fonction dans le texte. Il lit et relit le texte pour combler ce blanc. Le texte commence par la répétition de la même scène quotidienne de la famille trois fois consécutives, en changeant à chaque fois la structure de la phrase:

$$
\begin{aligned}
& \text { La mère fait du tricot } \\
& \text { Le fils fait la guerre } \\
& \text { (...) } \\
& \text { Et le père et le père } \\
& \text { (...) } \\
& \text { Il fait des affaires }
\end{aligned}
$$

Et la même scène se répète une deuxième fois ainsi:

Sa femme fait du tricot son fils la guerre

Lui des affaires

Et pour une troisième fois:

Le fils sa mère fait du tricot son père les

affaires et lui la guerre

Outre que la répétition de la même idée, nous trouvons dans ces vers précédents quil y a six mots remarquablement répétés: "mère", "père", "fils", "tricot", "guerre", et "affaires". Les trois premiers mots, qui appartiennent au même champ lexical, représentent trois membres de la famille qui doivent être solidement liés les uns aux autres. Pourtant, nous trouvons que ces mots sont séparés par les trois autres: tricot, guerre et affaires. Le tricot occupe la mère et la sépare de la famille, et c'est le même cas des affaires avec le père, et de la guerre avec le fils. Tandis que, paradoxalement, les trois mots (tricot, guerre, affaires) séparant les membres de la famille, et qui ne sont pas censés être liés les uns aux autres, à la fin du texte, nous les trouvons serrés, liés, semblant être, eux, d'une même famille:

$$
\begin{aligned}
& \text { La vie continue la vie avec le tricot la } \\
& \text { guerre les affaires } \\
& \text { Les affaires la guerre le tricot la guerre } \\
& \text { Les affaires les affaires et les affaires }
\end{aligned}
$$

A notre avis, le texte fait allusion aux relations fortes et entrelacées entre la guerre et les affaires, et nous découvrons ainsi que l'adjectif "familiale" utilisé dans le titre décrit cette relation. Ici encore, le contexte socioculturel où nous vivons influence notre interprétation du texte. Car, d'après nos expériences, nous savons que beaucoup de conflits armés et de scènes de violence que nous voyons tous les jours dans 
La Réception Visuelle du Texte: Analyse du Poème Familiale de Jacques Prévert Selon les Théories de la Réception

les quatre coins du monde ne sont que des conflits d'intérêts et d'affaires qui profitent à d'autres acteurs qui ne font pas partie du conflit.

C'est un indice très important offert par le texte que nous trouvons dans les trois derniers vers: le lecteur remarque que ce n'est pas une simple répétition et une simple insistance sur des mots, mais c'est une répétition accompagnée d'un changement de l'emplacement des mots, créant ainsi une sorte d'entrelacement entre les mots répétés, tout comme les fils qui s'entrelacent étroitement pour former un tissu de tricot, d'où le lien entre les mots "guerre", "affaires" et "tricot" dans le texte. C'est donc, comme nous avons vu selon Ricœur, la forme, la mise en ordre et l'agencement des éléments textuels qui nous ont aidés à cette interprétation du texte. Il convient de noter ici qu'à l'origine le mot "texte" est dérivé du latin "textus" qui signifie "tissu", du verbe Texere qui veut dire Tisser, etle texte "Familiale" nous le montre clairement. C'est le bon exemple d'un texte littéraire bien tissé, les mots y sont placés de manière qu'ils représentent des motifs de décoration d'un textile mais, en même temps, qui veulent donner un sens.

Dans cette étape de la lecture du texte, le rôle du lecteur est très important. Certes le texte donne ici des indices au lecteur, mais quel est le rôle du lecteur dans la compréhension du texte? Iser a parlé de l'interaction entre le texte et le lecteur, mais il n'a pas précisé le mécanisme de cette interaction, ce que nous essayerons d'expliquer dans les lignes suivantes: dans tout le texte Familiale, et surtout dans les derniers vers, ce qui aide le lecteur à comprendre le texte c'est le travail de son intelligence visuelle-spatiale. Cette intelligence, selon Gardner, est la capacité à créer des images mentales et les transformer. C'est aussi, entre autres, la capacité à saisir le sens des images et des peintures. Selon Gardner, la pensée, la réflexion et la résolution des problèmes passent toutes d'abord par l'utilisation des images mentales (Gardner 2011, 182 183).

Le texte, avec sa mise en forme, ses structures et son agencement des mots, stimule l'intelligence visuelle-spatiale chez le lecteur qui reçoit cette stimulation et utilise ses capacités visuelles pour interpréter le texte. Dans le cas du texte Familiale, la répétition des mots tricot, guerre, et affaires avec le changement de leur emplacement dans un ordre précis créent une forme significative dont le sens ne peut être saisi qu'avec la stimulation de l'intelligence visuelle-spatiale du lecteur. Cela met l'accent sur l'importance de la réception visuelle du texte.

\section{Conclusion}

Nous avons effectué une étude analytique du texte poétique intitulé "Familiale" selon les théories de la réception, en expliquant les étapes dans lesquelles notre lecture du texte est passée. A chaque étape, nous avons expliqué quel message du texte nous avons déduit et quelle théorie de la réception nous a aidés à justifier la conclusion de ce message. Ainsi, d'après notre interprétation, le texte "Familiale" veut transmettre deux messages principaux:

Premièrement, la guerre est devenue une routine quotidienne, à laquelle le peuple s'est habitué et face à laquelle il ne montre aucune réaction. Notre recours au contexte socioculturel dans lequel nous vivons et à notre expérience personnelle a grandement contribué à parvenir à cette conclusion, notamment pour 
expliquer le comportement des parents envers la participation de leur fils à la guerre et puis envers sa mort. Mais, ce qui avait évoqué ce contexte socioculturel dans notre réflexion c'est la rupture de notre horizon d'attente concernant le comportement naturel des parents dans une situation pareille.

Notre relation avec le texte pendant la lecture était, tout comme la décrit Iser, un processus d'interaction très efficace, qui s'est concrétisé à travers l'interrogation du texte en essayant de trouver des réponses aux questions, afin de remplir les blancs laissés par le texte. L'un des moyens que nous avons utilisés pour remplir ces blancs a été le recours à nos connaissances et lectures préalables pour expliquer la simplicité du langage dans le texte, qui nous a évoqué les comptines infantiles. Cette simplicité du langage symbolise la simplicité de penser la guerre dans la mentalité du peuple qui s'y est habitué.

En outre, nous avons essayé de prouver à travers cette analyse que l'interaction avec le texte exige du lecteur le travail de son intelligence visuelle afin de décrypter les indices que le texte lui offre pour le comprendre, ce qui a été le cas dans l'explication de l'abondance des répétitions dans le texte, surtout quand ces répétitions sont accompagnées du changement de l'emplacement des mots et des structures des phrases, créant ainsi une sorte d'entrelacement entre les phrases et les mots qui symbolise les relations entrelacées entre la guerre et les affaires. Alors, la relation entre le texte et le lecteur consiste, dans ce cas, en la stimulation par le texte de l'intelligence visuelle-spatiale du lecteur, et ce dernier reçoit cette stimulation et utilise ses capacités visuelles pour comprendre et interpréter le texte.

Enfin, il s'est avéré à travers cet article que les théories de la réception mentionnées dans notre étude contribuent toutes à effectuer une lecture complète et approfondie incluant tous les aspects littéraires et esthétiques du texte. 
La Réception Visuelle du Texte: Analyse du Poème Familiale de Jacques Prévert Selon les Théories de la Réception

$$
\begin{aligned}
& \text { التلقي البصري للنص:دراسة تحليلية لقصيدة (Familiale) لجاك بريفير } \\
& \text { حسب نظريات التلقي } \\
& \text { خالا يونس } \\
& \text { قسم اللفات الحديثة، جامعة اليرموك، الأردن ئن } \\
& \text { الملخص }
\end{aligned}
$$

تمثل هذه المقالة دراسة تحليلية لقصيدة (Familiale) حسب نظريات التلقي. تبين من خلال هذه الدراسة أن الفجوة

الجماليّة التي يخلقها النص تثد القارئ لاكتشاف أسرار هذا النص وتجذبه لقراءته وإعادة قراءته. ولا يقل تأثير السياق الثقافي

الاجتماعي للقارئ، من خلال إسقاط الأحداث والخبرات التي يعيشها القارئ في هذا السياق على النص، أهميةً وإسهاماً في عملية

فهم النص وتفسيره. وتكمن الأهمية الكبرى في العملية التفاعلية التبادلية بين النص والقارئ لتفسير النص وفهمه. إذ بينت هذه الدراسة أن جزءاً كبيراً من هذه العمليّة التفاعليّة تكمن في التلقي البصري للنص. حيث تكون العلاقة بين هذا الأخير والقارئ على شكل استثارة للذكاء البصري- المكاني لدى القارئ، ومن ثم يتم تلقي هذه الاستثارة من القارئ وتفعيل هذا الذكاء في عملية فهم النص وتفسيره. الكلمات المفتاحية: تحليل، تفسير، النص، الاستقبال، البصري. 


\section{Références}

Cools, Valérie. 2011. Le Lecteur en Théorie. Entre Plusieurs Modes De Pensée, in "Figures et Discours Critique", Figura, Centre de Recherche sur Le Texte et l'Imaginaire, coll. "Figura" (27), Montréal, Québec, Canada, 160p.

Fish, Stanley. 1980. Is There a Text in This Class? The Authority of Interpretive Communities, Harvard University Press, Cambridge

Gardner, Howard. 2011. Frames of Mind, the Theory of Multiple Intelligences, Basic Books, New York, première édition 1983.

Iser, Wolfgang. 1976. L'acte de Lecture. Théorie de L'effet Esthétique, Wavre, Mardaga, Coll. "Philosophie et langage", Bruxelles

Jauss, H. Robert. 1978. Pour une Esthétique de la Réception, Gallimard, coll. "Tel", Paris

Prévert, Jacques. 1949. Paroles, Gallimard, Paris, première édition 1946

Ricœur, Paul. 1986. Du Texte à l'Action. Essais d'Herméneutique II, Seuil, coll. "Essais", Paris 